\title{
The Number and Distinct Clustering Patterns of Voltage-Gated Calcium Channels in Nerve Terminals
}

\author{
Kohgaku Eguchi*, Jacqueline Montanaro, Elodie Le Monnier and Ryuichi Shigemoto* \\ Institute of Science and Technology Austria (IST Austria), Klosterneuburg, Austria
}

Upon the arrival of action potentials at nerve terminals, neurotransmitters are released from synaptic vesicles (SVs) by exocytosis. Cav2.1, 2.2, and 2.3 are the major subunits of the voltage-gated calcium channel (VGCC) responsible for increasing intraterminal calcium levels and triggering SV exocytosis in the central nervous system (CNS) synapses. The two-dimensional analysis of Cav2 distributions using sodium dodecyl sulfate (SDS)-digested freeze-fracture replica labeling (SDS-FRL) has revealed their numbers, densities, and nanoscale clustering patterns in individual presynaptic active zones. The variation in these properties affects the coupling of VGCCs with calcium sensors on SVs, synaptic efficacy, and temporal precision of transmission. In this study, we summarize how the morphological parameters of Cav2 distribution obtained using SDS-FRL differ depending on the different types of synapses and could correspond to functional properties in synaptic transmission.

Keywords: presynaptic active zone, synaptic vesicle, electron microscopy, freeze-fracture replica labeling, clustering analysis, voltage-gated calcium channel

\section{OPEN ACCESS}

Edited by:

Maria Eulalia Rubio,

University of Pittsburgh, United States

Reviewed by:

Timothy A. Ryan,

Cornell University, United States

*Correspondence:

Kohgaku Eguchi

kohgaku.eguchi@ist.ac.at

Ryuichi Shigemoto

ryuichi.shigemoto@ist.ac.at

Received: 31 December 2021

Accepted: 24 January 2022

Published: 24 February 2022

Citation:

Eguchi K, Montanaro J, Le Monnier E and Shigemoto $R$ (2022) The Number and Distinct Clustering Patterns of Voltage-Gated Calcium Channels in Nerve Terminals.

Front. Neuroanat. 16:846615. doi: 10.3389/fnana.2022.846615

\section{INTRODUCTION}

At a chemical synapse, the arrival of an action potential (AP) at the nerve terminal activates voltagegated calcium channels (VGCCs), thereby inducing $\mathrm{Ca}^{2+}$ influx into the terminal. Consequently, the fusion of synaptic vesicles (SVs) into the plasma membrane is triggered and neurotransmitters are released. VGCCs are composed of a pore-forming $\alpha 1$ subunit that is encoded by 10 genes and classified into three subgroups characterized with electrophysiological and pharmacological properties: $\mathrm{Ca}_{V} 1$ (L-type), $\mathrm{Ca}_{V} 2\left(\mathrm{~N}, \mathrm{P} / \mathrm{Q}\right.$, and R-type), and $\mathrm{Ca}_{V} 3$ (T-type) channels. The $\mathrm{Ca}_{V} 2$ channels $\left[\mathrm{Ca}_{V} 2.1\right.$ (P/Q-type), $\mathrm{Ca}_{V} 2.2$ (N-type), and $\mathrm{Ca}_{V} 2.3$ (R-type)] are highly expressed in chemical synapses of the mammalian central nervous system (CNS) and are concentrated at the presynaptic active zone (AZ). The AZ consists of protein complexes of various molecules including target soluble $\mathrm{N}$-ethylmaleimide-sensitive factor attached protein receptor (t-SNARE) proteins (syntaxin and SNAP-25) and scaffold proteins (e.g., RIMs, Munc-13, Bassoon, Piccolo, and ELKS) as well as VGCCs (Figure 1A). Fast and precise synaptic transmission is accomplished through the coupling of $\mathrm{Ca}_{V} 2$ channels and the $\mathrm{Ca}^{2+}$-sensor proteins (e.g., synaptotagmins) of releaseready docked SVs, which are adjacent to each other (Figure 1B). The issue of the required number of $\mathrm{Ca}_{V} 2$ channels for the fusion of a single SV is still under debate. Previous studies using squid giant synapses and chick ciliary ganglion calyx synapses have proposed that $\mathrm{Ca}^{2+}$ influx through a single VGCC can generate the fusion of an SV if the $\mathrm{Ca}^{2+}$ sensor is sufficiently close to the channel (Stanley, 2016). In chemical synapses of the mammalian CNS, however, $\mathrm{Ca}_{V} 2$ channels make clusters in the AZs, indicating that the $\mathrm{Ca}^{2+}$ influx through multiple $\mathrm{Ca}_{V} 2$ channels in a cluster generates the fusion of an SV. 
In this study, we have highlighted recent progress toward understanding the distribution and functions of $\mathrm{Ca}_{V} 2$ channels, mainly focusing on the findings based on an immunoelectron microscopic technique called sodium dodecyl sulfate-digested freeze-fracture replica labeling (SDS-FRL). SDS-FRL can be used to visualize the two-dimensional distribution of membrane molecules with high sensitivity and resolution (Masugi-Tokita et al., 2007) and is suitable for quantitative analysis of the number, density, and clustering of $\mathrm{Ca}_{V} 2$ channels in the AZs. Among the three $\mathrm{Ca}_{V} 2$ subunits, $\mathrm{Ca}_{V} 2.1$ has been most extensively examined by several groups and, thus, is mainly discussed here. We have also described the technical notes of SDS-FRL for accurate cluster analysis and have discussed the functional roles of $\mathrm{Ca}_{V} 2$ channel clustering on neurotransmitter release with different topographical models of $\mathrm{Ca}_{V} 2$ channels and SVs.

\section{OBSERVATION OF $\mathrm{Ca}_{v} 2$ CHANNEL DISTRIBUTION IN ACTIVE ZONES USING SODIUM DODECYL SULFATE-DIGESTED FREEZE-FRACTURE REPLICA LABELING}

Synaptic vesicles are thought to be docked near VGCCs in the AZs for the efficient triggering of their exocytosis in response to an AP. The geometric differences of $\mathrm{Ca}_{V} 2$ channels, such as their clustering and the distance from docked SVs, characterize the variety of the presynaptic properties. However, due to the high-density protein accumulation in the AZs, the accessibility of antibodies against $\mathrm{Ca}_{V} 2$ subtypes is potentially attenuated in the conventional immunofluorescence or pre-embedding immunoelectron microscopy. In contrast, the accessibility of the antibody to $\mathrm{Ca}_{V} 2$ channels is improved in SDS-FRL because the epitopes are exposed on the two-dimensional fractured face of the membrane after most of the cytosolic proteins that could mask the epitopes are washed out during the SDS treatment. However, SDS-FRL relies on random fracturing of frozen slices, making the identification of labeled profiles more difficult compared with volume data obtained by the threedimensional reconstruction of serial sections. SDS-FRL has been utilized for quantitative analysis of the nanoscale distribution of $\mathrm{Ca}_{V} 2$ channels at the AZs in rodent brains (Holderith et al., 2012; Indriati et al., 2013; Baur et al., 2015; Nakamura et al., 2015; Éltes et al., 2017; Kusch et al., 2018; Luján et al., 2018a,b; Rebola et al., 2019; Eguchi et al., 2020; Kleindienst et al., 2020; Bhandari et al., 2021). Figure 1C shows the examples of the replica labeling for $\mathrm{Ca}_{V} 2.1,2.2$, and 2.3 on presynaptic boutons in different synapses (Éltes et al., 2017; Bhandari et al., 2021). The quantitative analysis of the gold particle distribution suggests that all subtypes of $\mathrm{Ca}_{V} 2$ channels are accumulated in the AZs of presynaptic boutons. However, Cav2.3 has a high density in the surroundings of AZ too, which gradually decreases with distance from the AZ (Bhandari et al., 2021), whereas Cav2.1 shows very low density in the extra-AZ areas (Figure 1D).

\section{THE NUMBER AND DENSITY OF $\mathrm{Ca}_{v} 2$ CHANNELS IN INDIVIDUAL ACTIVE ZONES}

To estimate the absolute number of $\mathrm{Ca}_{V} 2$ channels, a one-toone relationship between single channels and gold particles in SDS-FRL would be ideal (labeling efficiency $=100 \%$ ). However, the immunogold labeling efficiency in replica samples can be attenuated or amplified for various reasons. Underestimation could occur because not all channel proteins are allocated to the protoplasmic (P-) or exoplasmic (E-) faces for the detection by primary antibodies against the intracellular or extracellular epitopes, respectively. Even if all epitopes are present on the P- or E-faces, not all may be bound to primary antibodies because of masking by associated proteins or low avidity of the antibodies. The immunolabeling conditions including antibody concentrations, temperature of SDS treatment, and contents of blocking and antibody incubation solutions should be optimized too (Kaufmann et al., 2021). In contrast, overestimation could occur because of the aggregation of primary or secondary antibodies or multiple binding of these antibodies to a single target (Figure 2A). In our previous studies, we calibrated the labeling efficiency of SDS-FRL with a $\mathrm{Ca}_{V} 2.1$ antibody in the cerebellum (Indriati et al., 2013; Miki et al., 2017) and calyx of Held (Nakamura et al., 2015), using the number of functional $\mathrm{P} / \mathrm{Q}$-type channels deduced by electrophysiological and $\mathrm{Ca}$ imaging data. However, some parameters used in the calibration were obtained in different species, ages, or brain regions. In addition, $\mathrm{Ca}^{2+}$ channels in the extra-AZ area were neglected. In this study, to estimate a more accurate labeling efficiency, we used $\mathrm{Ca}_{V} 2.1$ labeling in mossy fiber boutons (MFBs) of dentate gyrus granule cells (GCs) innerving CA3 pyramidal neurons (PNs) in the rat hippocampus. Previous electrophysiological and ultrastructural studies have reported the total number of $\mathrm{Ca}_{V} 2.1$ channels to be 1,320 per single MFB in rats at postnatal day (P) 21-22 (Li et al., 2007), and the total surface area of all AZs ( $\sim 30 \mathrm{AZs}$ in an MFB) and extra-AZ as 3.33 and $75.5 \mu \mathrm{m}^{2}$, respectively, in a single $\mathrm{MFB}$ of P28 rat (Rollenhagen et al., 2007). Using P28 rats, we labeled $\mathrm{Ca}_{V} 2.1$ in MFBs using an anti-Ca $_{V} 2.1$ antibody (Frontier Institute, Supplementary Table 1 and Figure 1C), which is essentially the same as those used in our previous studies (Indriati et al., 2013; Nakamura et al., 2015; Luján et al., 2018a,b; Eguchi et al., 2020). The AZs in the MFBs were automatically demarcated using a deep learningassisted software (Darea) with manual correction (Kleindienst et al., 2020) based on higher densities of intramembrane particles (IMPs) than extra-AZ on the P-face (Hagiwara et al., 2005; Figure 1D). The mean area of the complete AZs in the MFBs in replicas was $\sim 0.1 \mu \mathrm{m}^{2}$, which was close to the values obtained by the three-dimensional reconstructions of the MFBs (0.09$0.13 \mu \mathrm{m}^{2}$, Rollenhagen et al., 2007). The densities of gold particles labeling $\mathrm{Ca}_{V} 2.1$ in the AZs and extra-AZs were 232 and 0.84 particles/ $\mu \mathrm{m}^{2}$, respectively. This result and the area of AZ/extraAZ suggest that $92 \%$ of $\mathrm{Ca}_{V} 2.1$ channels are present in the AZs. Thus, the density of $\mathrm{Ca}_{V} 2.1$ at AZs is estimated to be $(1,320$ channels $\times 0.92) / 3.33 \mu \mathrm{m}^{2}=365$ channels $/ \mu \mathrm{m}^{2}$, and the $\mathrm{Ca}_{V} 2.1$ 
A

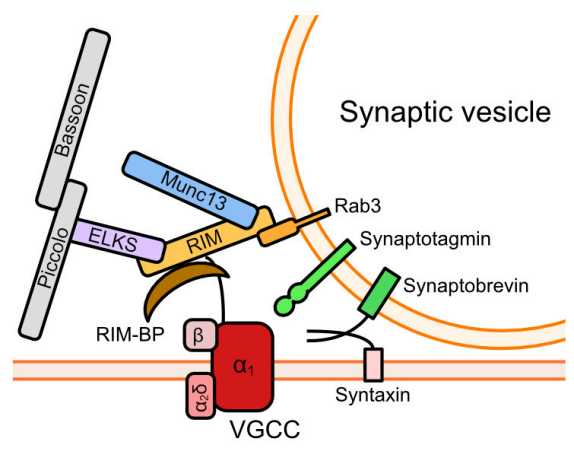

B

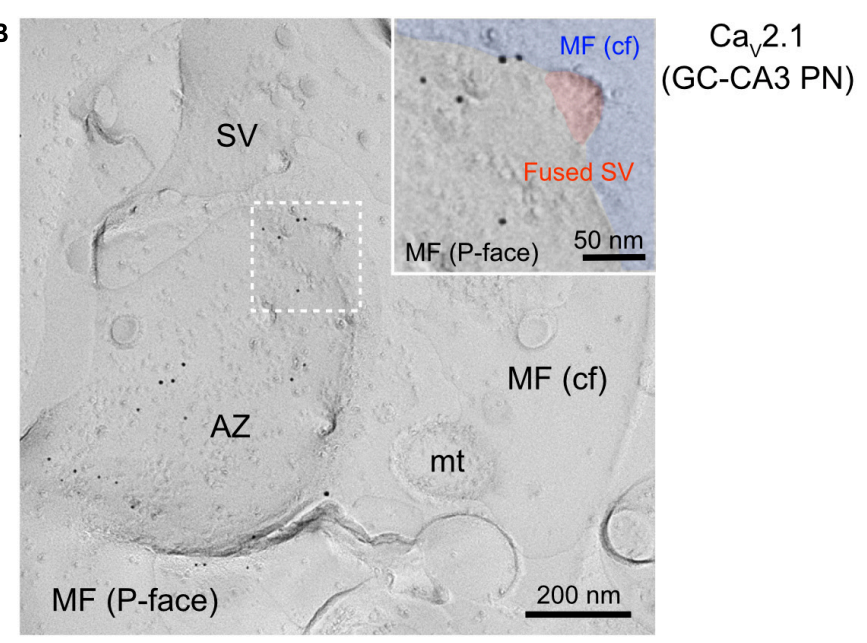

C

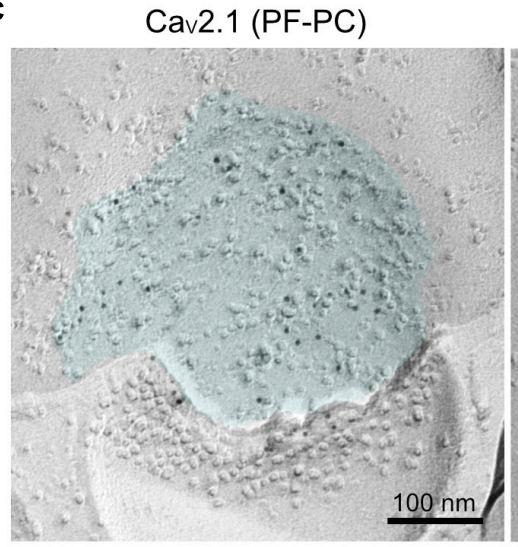

D

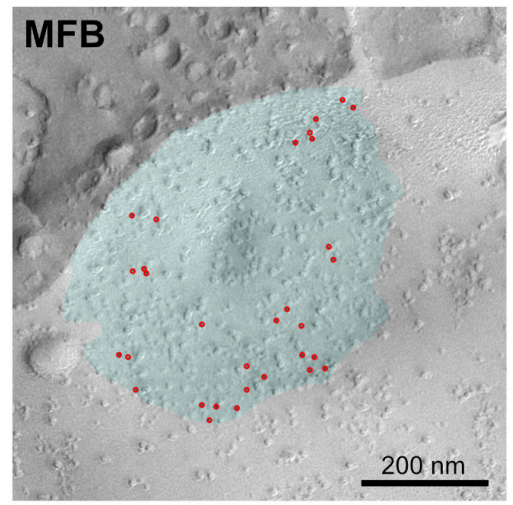

Cav2.2 (IN-CA3 PN)

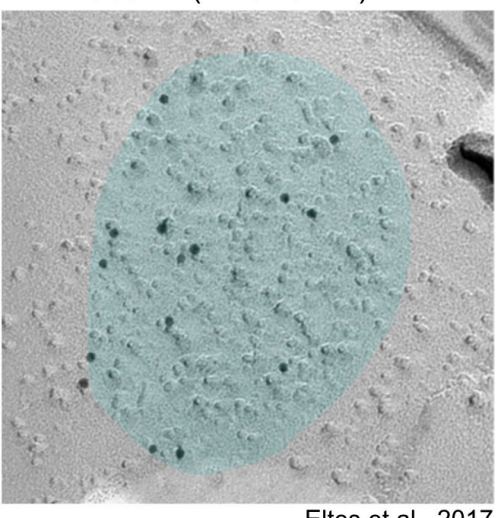

Eltes et al., 2017

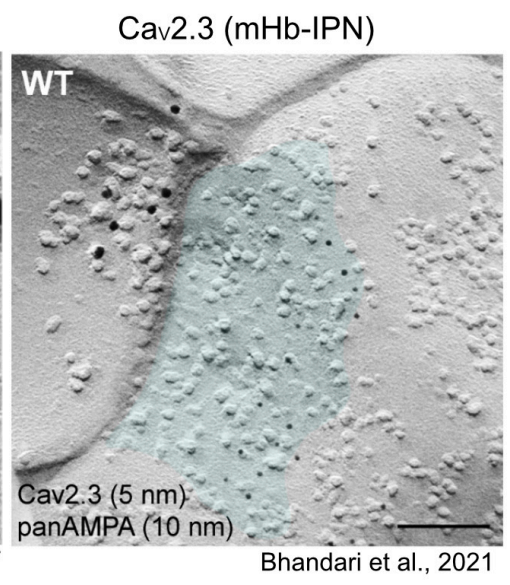

E

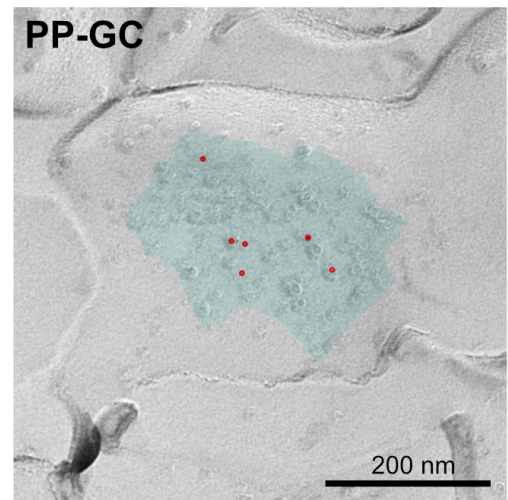

FIGURE 1 | Distribution of Cav2 channels at the presynaptic AZs. (A) A schema of a Cav2 channel assembled with presynaptic proteins related to SV fusion. (B) An example EM image of Cav2.1 particles concentrated in an AZ of a rat hippocampal dentate gyrus granule cell (GC)-CA3 pyramidal neuron (PN) synapse. Inset is a high-magnified image of the square area (dashed line) showing $\mathrm{Ca}_{V} 2.1$ particles around a fused SV (indicated with red). (C) Distribution of Cav2 subtypes in AZs of different synapse types. Left, labeling for Cav2.1 at a PF-PC synapse in the mouse cerebellum. Middle, labeling for Cav2.2 at an IN-CA3 PN synapse in the mouse hippocampus (Éltes et al., 2017). Right, labeling for Cav2.3 (5 nm) and AMPA-type glutamate receptor (panAMPA, $15 \mathrm{~nm}$ ) at an mHb-IPN synapse in the mouse brain (Bhandari et al., 2021). Cyan indicates AZ areas. (D) Left, Cav2.1 labeling at the AZ in an MFB of P28 Wistar rat. Red points indicate gold particles. Right, a bar graph showing the density of Cav2.1 particles in the AZs and non-AZ areas (non-AZs) of the rat MFBs. (E) Cav2.1 labeling in a perforant path-GC synapse obtained from the same replica preparation as panel (D). MF, mossy fiber; AZ, active zone; SV, synaptic vesicle; cf, cross-fracture; mt, mitochondria; PF, parallel fiber; PC,

Purkinje cell; IN, interneuron; mHb, medial habenula; IPN, interpeduncular nucleus; MFB, MF bouton. 
A

\section{Clustering of background particles? \\ Yes \\ $\downarrow$ No \\ Suitable for the cluster analysis}

\section{Aggregation of secondary $A B$ ?}

Dispersion on grid

No Use different secondary antibody

\section{Multiple seconday antibodies bound} to a primary $A B$ ?

Labeling of isolated target

No

$\checkmark$ Yes

\section{Aggregation of primary $A B$ ?}

HPLC

Protein aggregation detection reagents

$$
\downarrow \text { Yes }
$$

Use different primary antibody

Purify monomeric component

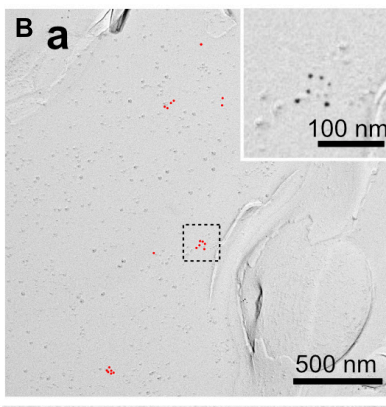

B b
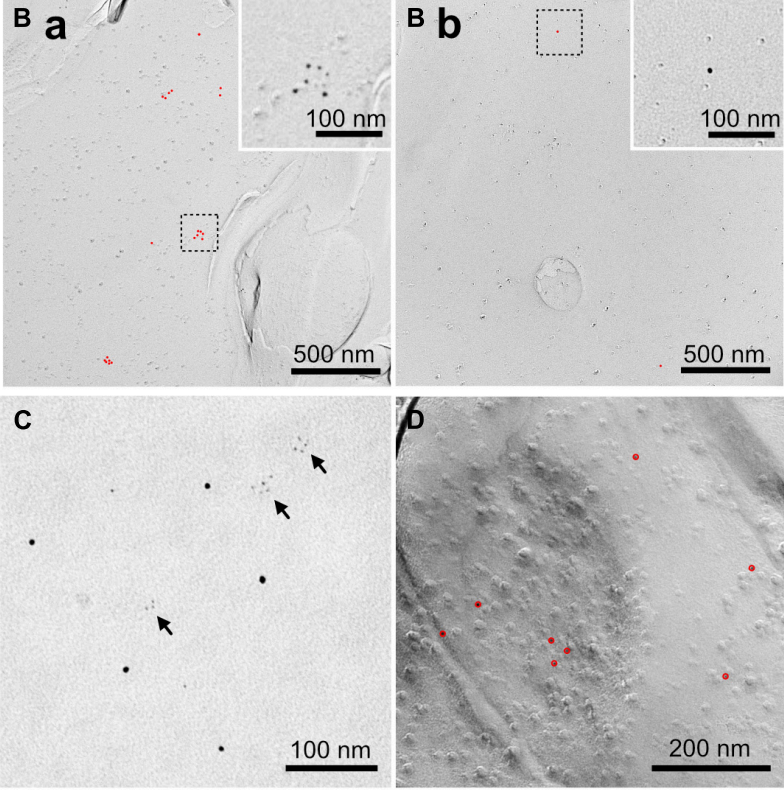

E
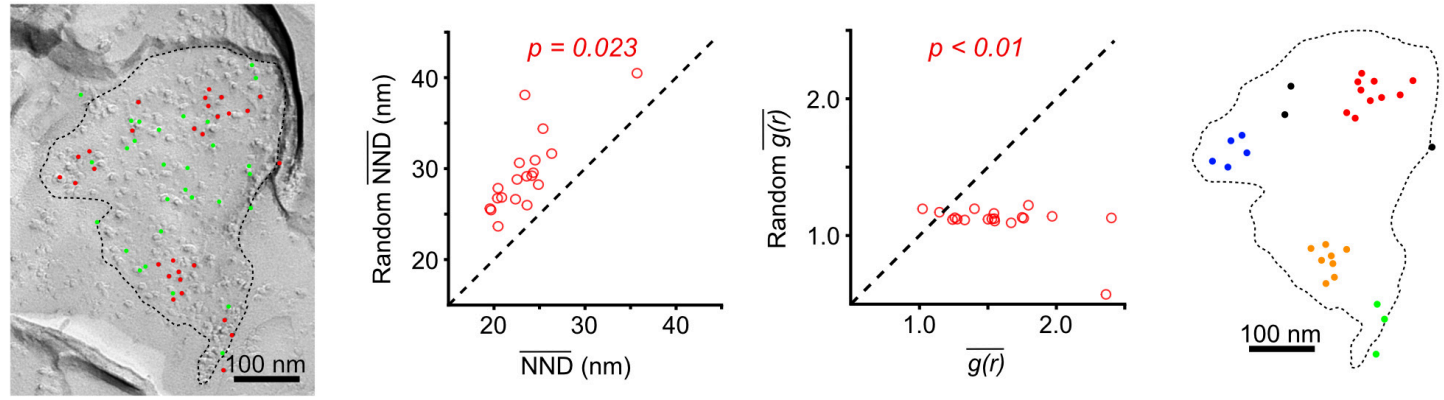

F

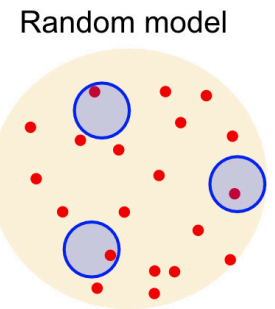

Perimeter release model

One-to-one

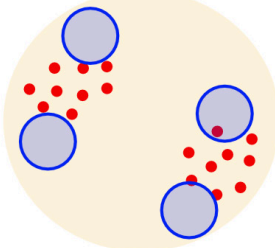
stoichiometry model

\section{Exclusion zone model}

Nakamura et al., 2015

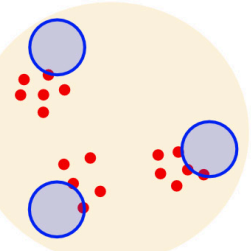

Miki et al., 2017

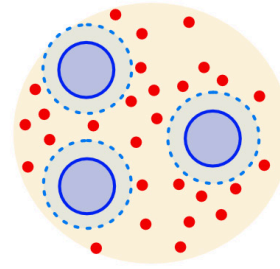

Rebola et al., 2019

FIGURE 2 | Cluster analysis of Cav2 channels and the distribution models. (A) A flowchart for checking the artificial clustering of gold particles on replica samples. (B) Clustering of gold particles with two different anti-Cav2.1 antibodies recognizing intracellular regions of Cav2.1, which should give specific labeling on the protoplasmic face (P-face) of the replica. These particles represent non-specific background because they are found on the exoplasmic face (E-face) of the dendritic plasma membrane. Antibody A showed non-specific labeling with clusters of several gold particles (Ba), whereas antibody B showed mostly isolated single background particles (Bb). (C) Aggregation of secondary antibodies. The EM image of a mixture of goat anti-rabbit lgG conjugated with 2 nm gold particle and goat anti-guinea pig lgG conjugated with $5 \mathrm{~nm}$ gold particle dispersed on a grid coated with formvar. The secondary antibody with $2 \mathrm{~nm}$ gold particle had aggregations (arrows), whereas that with $5 \mathrm{~nm}$ gold particle did not. (D) Sparse distribution of gold particles labeling VGluT1 at the PF bouton of the mouse cerebellum, indicating binding of single gold particle-conjugated secondary antibody to a primary antibody. Red circles indicate the gold particles. (E) Cluster analysis of Cav2.1. Left, an example of Cav2.1 labeling at a PF-PC synapse of the mouse cerebellum. Red and green points indicate real and randomly simulated particles, respectively, in the AZ (dashed line). Middle, comparison of the mean NND (left) and $g(r)$ values in each AZ obtained from the real and simulated particles. $P$-values were calculated by paired $t$-test. Right, cluster detection of the gold particles using DBSCAN with parameters of 3 for the minimum number of the particles in a cluster, and 
FIGURE 2 | mean NND + 2SD for the maximum distance $(\varepsilon)$ allowed for a particle to be included in a cluster. Colors indicate different clusters except for black indicating non-clustered particles. (F) Schema showing AZ topography models for Cav2 channels and release-ready docked SVs. Random model, random distribution of SVs and Cav2 channels. Perimeter release model, multiple SVs are positioned at the perimeter of a Cav2 channel cluster (Nakamura et al., 2015). One-to-one stoichiometry model, either 0 or 1 SV is docked per Cav 2 channel cluster (Miki et al., 2017). Exclusion zone model, Cav 2 channels are excluded from a zone around SVs (Rebola et al., 2019).

labeling efficiency is calculated to be $64 \%$. This value is very close to previously reported estimates using the same anti-Cav 2.1 antibody at rat cerebellar parallel fiber (PF)-Purkinje cell (PC) synapses (63\%, Indriati et al., 2013; 62\%, Miki et al., 2017) and at rat calyx of Held synapses (62\%, Nakamura et al., 2015). Using the same $\mathrm{Ca}_{V} 2.1$ antibody and similar labeling conditions, we compared the estimated number and density of $\mathrm{Ca}_{V} 2.1$ channels in different types of CNS synapses (Supplementary Table 2). In the hippocampus, three types of excitatory synapses showed a range of 9.8-24.2 channels as per AZ but with similar densities (288-358 channels $/ \mu \mathrm{m}^{2}$ ) and nearest neighbor distances (NND, an index for local density, $27-29 \mathrm{~nm}$ ), indicating that the difference in number is mostly ascribable to that in the $\mathrm{AZ}$ size. Interestingly, the center-periphery index (CPI, Kleindienst et al., 2020) showed a significantly lower value $(P<0.0001)$ in perforant path-to-granule cell (PP-GC) synapses (0.42) than that in MFB synapses (0.67), indicating that $\mathrm{Ca}_{V} 2.1$ is localized nearer to the AZ center in PP-GC synapses (Figures 1D,E). In the cerebellum, the $\mathrm{Ca}_{V} 2.1$ density in PF boutons was higher in synapses made on molecular layer interneurons (MLI, 827 channels $\left./ \mu \mathrm{m}^{2}\right)$ than those on PCs $\left(416\right.$ channels $\left./ \mu \mathrm{m}^{2}\right)$, indicating target cell-dependent regulation of $\mathrm{Ca}_{V} 2.1$ density. Although the Cav2.1 numbers are linearly correlated with release probability in excitatory synapses of CA3 hippocampal pyramidal cells (Holderith et al., 2012), the number or density does not necessarily correlate with release probability or $\mathrm{Ca}^{2+}$ influx across different types of synapses (Éltes et al., 2017; Rebola et al., 2019). Functional properties of single channels or spatial relationship between $\mathrm{Ca}^{2+}$ channel clusters and release-ready docked SVs should also affect the synaptic efficacy and temporal precision of neurotransmission.

\section{TECHNICAL CONSIDERATION OF CLUSTERING ANALYSIS USING SODIUM DODECYL SULFATE-DIGESTED FREEZE-FRACTURE REPLICA LABELING}

Previous studies have demonstrated not only the number/density of $\mathrm{Ca}_{V} 2.1$ channels in the AZs but also their clustering (Indriati et al., 2013; Nakamura et al., 2015; Miki et al., 2017; Rebola et al., 2019; Kleindienst et al., 2020), indicating the massive influx of $\mathrm{Ca}^{2+}$ through the clustered channels to effectively increase the $\mathrm{Ca}^{2+}$ level at the nano-spots in the AZs. To estimate clustering patterns accurately, a high labeling efficiency as described earlier is desirable. Moreover, some technical pitfalls causing artificial gold particle clustering must be excluded (Figure 2A). A valuable indicator of the artificial cluster formation is the non-specific background labeling of gold particles on the "wrong" face or cross-fracture (Figure 2B). In the case of our $\mathrm{Ca}_{V} 2.1$ labeling with the antibody against an intracellular epitope, gold particles on the E-face could serve as background labeling. Figure 2B shows examples of such non-specific labeling with different anti$\mathrm{Ca}_{V} 2.1$ antibodies on the dendritic E-face in mouse cerebellar PCs. The gold particles with antibody A were clustered with 46 particles, whereas those with antibody B were mostly isolated single particles. If the background gold particles make clusters, the results of clustering analysis for the specific labeling could be misleading. Possible causes of this artificial clustering can be examined and eliminated as follows (Figure 2A).

\section{Aggregation of Antibodies}

Immunoglobulin Gs (IgGs) are known to form aggregations due to various reasons, including acidic $\mathrm{pH}$, often used for purification (Lopez et al., 2019). If the gold-conjugated secondary antibodies are aggregated, they make artificial clusters on the replica. The aggregation of secondary antibodies can be easily tested by observing the antibodies dispersed on a grid coated with formvar under electron microscope (EM) (Figure 2C). In this example, anti-rabbit IgG conjugated with $2 \mathrm{~nm}$ but not anti-guinea pig IgG conjugated with $5 \mathrm{~nm}$ gold particles formed clusters due to the antibody aggregation. This test also excludes the conjugation of multiple gold particles to a single secondary antibody. Checking the aggregation of primary antibodies is more time-consuming because it cannot be observed directly under EM unless they are negatively stained. Several analytical chemistry techniques such as high-performance liquid chromatography (HPLC) and fluorescent dyes for detecting aggregated proteins are suitable for detecting primary antibody aggregation (Liu et al., 2016; Oshinbolu et al., 2018). HPLC can be also used to purify the monomeric component of the primary antibody.

\section{Multiple Binding of Antibodies to a Single Protein}

In immunofluorescence methods, the signal is enhanced when multiple secondary antibodies conjugated with fluorescent dyes bind to a single primary antibody. However, such a signal amplification hampers clustering analysis in SDS-FRL. To examine the possibility of this type of multiple particle binding to a single target, we labeled vesicular glutamate transporter 1 (VGluT1), which is an SV protein that is sparsely distributed on the PF bouton membrane in the mouse cerebellum (Miki et al., 2017). The $5 \mathrm{~nm}$ gold particles conjugated with antiguinea pig IgG (the same one as we verified earlier) were sparsely distributed without clustering, indicating no multiple binding of the secondary antibody to a single primary antibody (Figure 2D). If all the possible reasons for artificial signal amplification described above are excluded, the last possibility 
is the multiple binding of primary antibodies to a single target protein. Although it should not often occur, since epitope regions in a single target protein are usually much smaller than the primary antibody, the use of monoclonal primary antibodies would help to avoid this problem.

\section{CLUSTER ANALYSIS OF Cav2.1 CHANNELS AT ACTIVE ZONES}

A great variety of methods have been developed to detect particle clusters by comparison with random distributions; Szoboszlay et al. (2017) evaluated the benefits and effectiveness of the implemented methods, and they recommended the comparison of the mean NND between particles as a method to investigate whether gold particles form uniform or clustered patterns compared with random point patterns. If the particles are clustered, the mean NND will be significantly smaller than that of randomly distributed particles computed from Monte Carlo simulations. They also recommended the spatial autocorrelation function (ACF), described as $g(r)$, a derivative of Ripley's $K$ function based on all-to-all distances between particles, as another useful method to detect clustering. $g(r)$ values close to 1 indicate the random distribution of particles, values $<1$ indicate a uniform distribution, and values $>1$ indicate clustering. In Figure 2E, we analyzed the $\mathrm{Ca}_{V} 2.1$ clustering in the AZs at PFPC synapses of the mouse cerebellum. We calculated the mean values of NND and $g(r)$ of the real particles distributed within the AZs on the replicas and the simulated particles with random distribution (200 times simulations for each examined AZ). The mean values of real NND and $g(r)$ were significantly smaller and larger, respectively, than their corresponding simulated values $(P<0.05$, paired $t$-test, $n=20 \mathrm{AZs})$, suggesting that $\mathrm{Ca}_{V} 2.1$ is distributed in a clustered manner within these AZs.

Several methods have been utilized to automatically detect each cluster of gold particles on the replica. A simple way to define a cluster is to consider particles that exist within a radius (e.g., $100 \mathrm{~nm}$ ) of each particle to compose a cluster (Nakamura et al., 2015). An advanced method is density-based spatial clustering of applications with noise (DBSCAN), which is a density-based clustering algorithm that groups together points that are tightly clustered while marking points that are less dense and singly distributed as outliers (Szoboszlay et al., 2017). Figure 2E demonstrates the cluster analysis of $\mathrm{Ca}_{V} 2.1$ immunolabeled AZs using DBSCAN with two parameters: the radius of a neighborhood (i.e., the maximum distance between two particles for one to be considered as in the neighborhood of the other) as mean NND plus two times its SD and the minimum number of particles necessary to form a cluster as 3. The DBSCAN algorithm detected $2.6 \pm 0.2$ clusters in PFPC synapses $(n=65 \mathrm{AZs})$. The gold particle clusters consist of $6.8 \pm 0.3$ particles $(n=167$ clusters), indicating that $10.6 \pm 0.5$ channels form a cluster in the AZs estimated with the labeling efficiency (64\%).

Supplementary Table 2 shows the summary of the $\mathrm{Ca}_{V} 2.1$ channel clustering, which was detected in all types of rodent CNS synapses analyzed so far in the previous and present studies using the same primary antibody (refer to the section
"Materials and Methods" and Supplementary Table 1). Although the number and density of clusters vary depending on the synapse types, the mean NNDs and numbers of particles per cluster were quite similar, indicating a common mechanism of $\mathrm{Ca}_{V} 2.1$ cluster formation. The distribution of $\mathrm{Ca}_{V} 2.1$ clusters should be examined relative to docked SVs distribution for considering their functional implications (Nakamura et al., 2015). Several different models of the topographical relationship between $\mathrm{Ca}_{V} 2$ channel clusters and docked SVs have been proposed as discussed in the following section (Figure 2F).

\section{TOPOGRAPHICAL MODELS OF Cav2 CLUSTERING AND SYNAPTIC VESICLE FUSION}

Voltage-gated calcium channels in the AZs are coupled with calcium sensors, synaptotagmins, on the docked SVs to efficiently evoke neurotransmitter release. Although it is still being debated about how many VGCCs are required to evoke a vesicle fusion, SDS-FRL observations have provided crucial information to understand the contribution of VGCC distribution to neurotransmitter release. At calyx of Held synapses in the rat auditory brainstem, the number of $\mathrm{Ca}_{V} 2.1$ in clusters increased during hearing onset (P7 vs. P14) with no changes in NND, and the cluster area was expanded (Nakamura et al., 2015). This developmental change results in tighter coupling of $\mathrm{Ca}_{V} 2.1$ channel clusters with vesicle fusion sites. A computational simulation based on this as well as electrophysiological and $\mathrm{Ca}^{2+}$ imaging results predicts that multiple docked SVs are located at the perimeter of a $\mathrm{Ca}_{V} 2.1$ cluster, and the $\mathrm{Ca}_{V} 2.1$ number in a cluster mainly determines the vesicular release probability ("perimeter release model," Figure 2F). In contrast, at PF-MLI synapses of the mouse cerebellar cortex, NNDs between particles were significantly shortened during postnatal development (postnatal week 2 vs. 4) without changes of $\mathrm{Ca}_{V} 2.1$ number in the cluster (Miki et al., 2017). This tighter VGCC arrangement may allow for more efficient coupling between $\mathrm{Ca}^{2+}$ entry and SV release. Interestingly, the numbers of SV docking sites (estimated from electrophysiological experiments) and $\mathrm{Ca}_{V} 2.1$ clusters showed a close correspondence, leading to propose "one-to-one stoichiometry model" (Figure 2F). In contrast, Rebola et al. (2019) proposed that $\mathrm{Ca}_{V} 2.1$ channels were not clustered but simply excluded from a $50 \mathrm{~nm}$ zone around docked SVs (“exclusion zone model," Figure 2F) at cerebellar PF-PC synapses. However, this contrasts with observations in GABAergic stellate cell synapses, where SVs are tightly associated $(\sim 10 \mathrm{~nm})$ with the perimeter of VGCC clusters consistent with the perimeter release model (Rebola et al., 2019). In the exclusion zone model, the release probability is determined by the radius of the exclusion zones rather than the number of $\mathrm{Ca}_{V} 2.1$ in the AZs.

\section{CONCLUSION}

Sodium dodecyl sulfate-digested FRL studies demonstrate the nanoscale distribution of $\mathrm{Ca}_{V} 2$ channels at AZs of presynaptic terminals. Although a single VGCC can generate the fusion 
of a single SV (Stanley, 2016), $\mathrm{Ca}_{V} 2$ channels form clusters (approximately 10 channels) in the AZs of many synapse types in the CNS. The distribution of $\mathrm{Ca}_{V} 2$ channels, especially topographical relationships between $\mathrm{Ca}_{V} 2$ channel clusters and $\mathrm{Ca}^{2+}$ sensors of docked SVs, and the number of channels in a cluster modulate the properties of neurotransmitter release. The dynamic changes in the $\mathrm{Ca}_{V} 2$ channel clustering might contribute to the presynaptic plasticity of synaptic transmission. It remains unclear how the distribution of $\mathrm{Ca}_{V} 2$ channels and their topographical relationships with SVs are regulated in different types of synapses. The quantitative nanoscale analysis of $\mathrm{Ca}_{V} 2$ channel distribution by SDS-FRL, combined with simultaneous visualization of the release-ready docked SVs, should be useful to unveil these questions.

\section{DATA AVAILABILITY STATEMENT}

The raw data supporting the conclusions of this article will be made available by the authors, without undue reservation.

\section{ETHICS STATEMENT}

The animal study was reviewed and approved by the Ethics Committee of IST Austria.

\section{REFERENCES}

Baur, D., Bornschein, G., Althof, D., Watanabe, M., Kulik, A., Eilers, J., et al. (2015). Developmental tightening of cerebellar cortical synaptic influx-release coupling. J. Neurosci. 35, 1858-1871. doi: 10.1523/JNEUROSCI.2900-14.2015

Bhandari, P., Vandael, D., Fernández-Fernández, D., Fritzius, T., Kleindienst, D., Önal, C., et al. (2021). GABAB receptor auxiliary subunits modulate Cav2.3mediated release from medial habenula terminals. Elife 10:e68274. doi: 10.7554/ eLife. 68274

Eguchi, K., Velicky, P., Hollergschwandtner, E., Itakura, M., Fukazawa, Y., Danzl, J. G., et al. (2020). Advantages of Acute Brain Slices Prepared at Physiological Temperature in the Characterization of Synaptic Functions. Front. Cell. Neurosci. 14:63. doi: 10.3389/fncel.2020.00063

Éltes, T., Kirizs, T., Nusser, Z., and Holderith, N. (2017). Target Cell TypeDependent Differences in Ca2+ Channel Function Underlie Distinct Release Probabilities at Hippocampal Glutamatergic Terminals. J. Neurosci. 37, 19101924. doi: 10.1523/JNEUROSCI.2024-16.2017

Hagiwara, A., Fukazawa, Y., Deguchi-Tawarada, M., Ohtsuka, T., and Shigemoto, R. (2005). Differential distribution of release-related proteins in the hippocampal CA3 area as revealed by freeze-fracture replica labeling. J. Comp. Neurol. 489, 195-216. doi: 10.1002/cne.20633

Holderith, N., Lorincz, A., Katona, G., Rózsa, B., Kulik, A., Watanabe, M., et al. (2012). Release probability of hippocampal glutamatergic terminals scales with the size of the active zone. Nat. Neurosci. 15, 988-997. doi: 10.1038/nn.3137

Indriati, D. W., Kamasawa, N., Matsui, K., Meredith, A. L., Watanabe, M., and Shigemoto, R. (2013). Quantitative localization of Cav2.1 (P/Q-type) voltage-dependent calcium channels in Purkinje cells: somatodendritic gradient and distinct somatic coclustering with calcium-activated potassium channels. J. Neurosci. 33, 3668-3678. doi: 10.1523/JNEUROSCI.2921-12.2013

Kaufmann, W. A., Kleindienst, D., Harada, H., and Shigemoto, R. (2021). Highresolution localization and quantitation of membrane proteins by SDS-digested freeze-fracture replica labeling (SDS-FRL)," in Neuromethods Neuromethods. New York, NY: Springer US, 267-283.

Kleindienst, D., Montanaro, J., Bhandari, P., Case, M. J., Fukazawa, Y., and Shigemoto, R. (2020). Deep Learning-Assisted High-Throughput Analysis of

\section{AUTHOR CONTRIBUTIONS}

KE and RS conceived and wrote the manuscript. All authors conducted electron microscopic experiments and contributed to the article and approved the submitted version.

\section{FUNDING}

This work was supported by the European Research Council advanced grant No. 694539 and the joint German-Austrian DFG and FWF project SYNABS (FWF: I-4638-B) to RS.

\section{ACKNOWLEDGMENTS}

The authors thank Walter Kaufmann for his critical comments on the manuscript.

\section{SUPPLEMENTARY MATERIAL}

The Supplementary Material for this article can be found online at: https://www.frontiersin.org/articles/10.3389/fnana. 2022.846615/full\#supplementary-material

Freeze-Fracture Replica Images Applied to Glutamate Receptors and Calcium Channels at Hippocampal Synapses. Int. J. Mol. Sci. 21:6737. doi: 10.3390/ ijms21186737

Kusch, V., Bornschein, G., Loreth, D., Bank, J., Jordan, J., Baur, D., et al. (2018). Munc13-3 Is Required for the Developmental Localization of Ca2+ Channels to Active Zones and the Nanopositioning of Cav2.1 Near Release Sensors. Cell Rep. 22, 1965-1973. doi: 10.1016/j.celrep.2018.0 2.010

Li, L., Bischofberger, J., and Jonas, P. (2007). Differential gating and recruitment of P/ Q-, N-, and R-type Ca2+ channels in hippocampal mossy fiber boutons. J. Neurosci. 27, 13420-13429. doi: 10.1523/JNEUROSCI.1709-07. 2007

Liu, B., Guo, H., Xu, J., Qin, T., Xu, L., Zhang, J., et al. (2016). Acid-induced aggregation propensity of nivolumab is dependent on the Fc. MAbs 8, 110711170. doi: 10.1080/19420862.2016.1197443

Lopez, E., Scott, N. E., Wines, B. D., Hogarth, P. M., Wheatley, A. K., Kent, S. J., et al. (2019). Low pH Exposure During Immunoglobulin G Purification Methods Results in Aggregates That Avidly Bind Fc $\gamma$ Receptors: implications for Measuring Fc Dependent Antibody Functions. Front. Immunol. 10:2415. doi: 10.3389/fimmu.2019.02415

Luján, R., Aguado, C., Ciruela, F., Arus, X. M., Martín-Belmonte, A., Alfaro-Ruiz, R., et al. (2018a). SK2 Channels Associate With mGlu1 $\alpha$ Receptors and CaV2.1 Channels in Purkinje Cells. Front. Cell. Neurosci. 12:311. doi: 10.3389/fncel. 2018.00311

Luján, R., Aguado, C., Ciruela, F., Cózar, J., Kleindienst, D., de la Ossa, L., et al. (2018b). Differential association of GABAB receptors with their effector ion channels in Purkinje cells. Brain Struct. Funct. 223, 1565-1587. doi: 10.1007/ s00429-017-1568-y

Masugi-Tokita, M., Tarusawa, E., Watanabe, M., Molnar, E., Fujimoto, K., and Shigemoto, R. (2007). Number and Density of AMPA Receptors in Individual Synapses in the Rat Cerebellum as Revealed by SDS-Digested Freeze-Fracture Replica Labeling. J. Neurosci. 27, 2135-2144. doi: 10.1523/JNEUROSCI.286106.2007

Miki, T., Kaufmann, W. A., Malagon, G., Gomez, L., Tabuchi, K., Watanabe, M., et al. (2017). ). Numbers of presynaptic Ca 2+ channel clusters 
match those of functionally defined vesicular docking sites in single central synapses. Proc. Natl. Acad. Sci. 114, E5246-E5255. doi: 10.1073/pnas.170447 0114

Nakamura, Y., Harada, H., Kamasawa, N., Matsui, K., Rothman, J. S., Shigemoto, R., et al. (2015). Nanoscale Distribution of Presynaptic Ca2+Channels and Its Impact on Vesicular Release during Development. Neuron 85, 145-159. doi: 10.1016/j.neuron.2014.11.019

Oshinbolu, S., Shah, R., Finka, G., Molloy, M., Uden, M., and Bracewell, D. G. (2018). Evaluation of fluorescent dyes to measure protein aggregation within mammalian cell culture supernatants. J. Chem. Technol. Biotechnol. 93, 909917. doi: $10.1002 /$ jctb.5519

Rebola, N., Reva, M., Kirizs, T., Szoboszlay, M., Lõrincz, A., Moneron, G., et al. (2019). Distinct Nanoscale Calcium Channel and Synaptic Vesicle Topographies Contribute to the Diversity of Synaptic Function. Neuron 104, 693-710.e9.

Rollenhagen, A., Sätzler, K., Rodríguez, E. P., Jonas, P., Frotscher, M., and Lübke, J. H. R. (2007). Structural determinants of transmission at large hippocampal mossy fiber synapses. J. Neurosci. 27, 10434-10444. doi: 10.1523/JNEUROSCI. 1946-07.2007

Stanley, E. F. (2016). The Nanophysiology of Fast Transmitter Release. Trends Neurosci. 39, 183-197. doi: 10.1016/j.tins.2016.01.005
Szoboszlay, M., Kirizs, T., and Nusser, Z. (2017). Objective quantification of nanoscale protein distributions. Sci. Rep. 7:15240. doi: 10.1038/s41598-017$15695-\mathrm{w}$

Conflict of Interest: The authors declare that the research was conducted in the absence of any commercial or financial relationships that could be construed as a potential conflict of interest.

Publisher's Note: All claims expressed in this article are solely those of the authors and do not necessarily represent those of their affiliated organizations, or those of the publisher, the editors and the reviewers. Any product that may be evaluated in this article, or claim that may be made by its manufacturer, is not guaranteed or endorsed by the publisher.

Copyright ( 2022 Eguchi, Montanaro, Le Monnier and Shigemoto. This is an openaccess article distributed under the terms of the Creative Commons Attribution License (CC BY). The use, distribution or reproduction in other forums is permitted, provided the original author(s) and the copyright owner(s) are credited and that the original publication in this journal is cited, in accordance with accepted academic practice. No use, distribution or reproduction is permitted which does not comply with these terms. 